\title{
Renal Protective Effect of DPP-4 Inhibitors in Type 2 Diabetes Mellitus Patients: A Cohort Study
}

\author{
Young-Gun Kim, ${ }^{1}$ JungHyun Byun,, Dukyong Yoon, ${ }^{1}$ Ja Young Jeon, ${ }^{2}$ Seung Jin Han, \\ Dae Jung Kim, ${ }^{2}$ Kwan-Woo Lee, ${ }^{2}$ Rae Woong Park, ${ }^{1,3}$ and Hae Jin Kim ${ }^{2}$ \\ ${ }^{1}$ Department of Biomedical Informatics, Ajou University School of Medicine, Suwon, Republic of Korea \\ ${ }^{2}$ Department of Endocrinology and Metabolism, Ajou University School of Medicine, Suwon, Republic of Korea \\ ${ }^{3}$ Department of Biomedical Sciences, Ajou University Graduate School of Medicine, Suwon, Republic of Korea \\ Correspondence should be addressed to Rae Woong Park; veritas@ajou.ac.kr and Hae Jin Kim; jinkim@ajou.ac.kr
}

Received 19 October 2016; Revised 1 December 2016; Accepted 12 December 2016

Academic Editor: Feng Wang

Copyright (C) 2016 Young-Gun Kim et al. This is an open access article distributed under the Creative Commons Attribution License, which permits unrestricted use, distribution, and reproduction in any medium, provided the original work is properly cited.

\begin{abstract}
Aims. Dipeptidyl-peptidase IV inhibitors (DPP-4i) are among the most popular oral antidiabetic agents. However, the effects of DPP-4i on diabetic nephropathy are not well-established. The aim of this study was to determine the renoprotective effects of DPP-4i, using albuminuria and glomerular filtration rate (GFR) as indicators, in type 2 diabetes mellitus (T2DM) patients. Methods. This retrospective observational cohort study used the clinical database of a tertiary hospital. The changes of urine albumin/creatinine ratio (UACR), estimated GFR (eGFR), and metabolic parameters after treatment were compared with the changes of those parameters before treatment using paired Student's $t$-test. Results. The mean UACR in the entire study population decreased to approximately $45 \mathrm{mg} / \mathrm{g} 1$ year after DPP- $4 \mathrm{i}$ treatment, while it was increased approximately $39 \mathrm{mg} / \mathrm{g} 1$ year before DPP-4i treatment $(p<0.05)$. Patients with macroalbuminuria showed a significant reduction in albumin levels after DPP-4i treatment $(p<0.05)$; however, patients with microalbuminuria and normoalbuminuria did not show improvements in albuminuria levels after treatment. Although eGFR was not changed 1 year after DPP-4i treatment, reductions in eGFR were slowed in patients with microalbuminuria and reversed in the macroalbuminuria or normoalbuminuria groups, 4 years after treatment. Conclusions. Administration of DPP-4i reduces urine albumin excretion and mitigates reduction of eGFR in T2DM patients.
\end{abstract}

\section{Introduction}

Diabetic nephropathy is one of the most important complications of diabetes, being strongly associated with increased overall mortality, cardiovascular mortality, cardiovascular events, and end-stage renal disease $[1,2]$. The major and earliest clinical manifestation of diabetic nephropathy is albuminuria. Albuminuria is a principal marker of kidney damage, which is caused by glomerular leakage. Generally, albuminuria is used as a marker of diabetic nephropathy; many studies have suggested that albuminuria is correlated with the progression of diabetic nephropathy, cardiovascular mortality, and all-cause mortality [3-6]. In addition, reduction of albuminuria with drugs is associated with renal protection [7-9]. However, some studies have suggested that albuminuria is not an appropriate therapeutic target for diabetic nephropathy [10]. Indeed, both loop and thiazide diuretics resulted in a reduction of albuminuria but did not improve renal outcome $[11,12]$. For this reason, albuminuria and glomerular filtration rate (GFR) should be considered together as surrogate markers for diabetic nephropathy.

Dipeptidyl-peptidase IV inhibitors (DPP-4i) are among the most popular and effective oral antidiabetic agents. They have many advantages, including high glucose-lowering potency, low risk of hypoglycemia, no association with weight gain, and being tolerable in chronic renal failure patients. However, their efficacy for preventing diabetic complications, especially diabetic nephropathy, is not wellestablished. Physiologically, dipeptidyl-peptidase IV (DPP4) acts on nephrons to exert various functions [13, 14], and some preclinical studies have suggested that DPP-4i exerts renoprotective effects [15-17]. However, clinical evidence 
regarding the renal protective effects of DPP-4i therapy is limited.

The majority of clinical studies investigating the renoprotective effects of DPP-4i have only focused on evaluating their albuminuria-lowering ability $[18,19]$. Although several studies have been proposed on this topic, it is still unclear that DPP-4i slows the deterioration of GFR in diabetic nephropathy [20, 21]. Moreover, almost all of these studies only contained short-term follow-up data [18-22] and included only one DPP-4i drug; thus, they were unable to evaluate effects according to drug class.

The current study aimed to determine the renoprotective effects of DPP-4i, using albuminuria and GFR as indicators, in type 2 diabetes mellitus (T2DM) patients.

\section{Methods}

2.1. Study Design and Subjects. A retrospective observational cohort study was conducted using the clinical database of Ajou University Hospital, which is a South Korean tertiary hospital with 1,108 beds. The study protocol was approved by the Institutional Review Board of Ajou University Hospital.

The inclusion criteria for study participants were as follows: (1) aged $\geq 19$ years with T2DM identified by the International Classification of Disease, Tenth Revision code E11; (2) prescribed DPP-4i from March 1, 2010, to February 28, 2014; and (3) data on urine albumin and creatinine levels at baseline, 1 year prior to starting DPP-4i, and 1 year after DPP-4i treatment initiation.

Exclusion criteria were as follows: glycosylated hemoglobin $(\mathrm{HbAlc}) \leq 6.5 \%(48 \mathrm{mmol} / \mathrm{mol})$ or $>10 \%(86 \mathrm{mmol} / \mathrm{mol})$; baseline estimated GFR (eGFR) $\leq 15 \mathrm{~mL} / \mathrm{min} / 1.73 \mathrm{~m}^{2}$; currently undergoing dialysis; body mass index (BMI) > $40 \mathrm{~kg} / \mathrm{m}^{2}$; treatment with insulin; treatment with steroids for $>7$ days; and patients with dual blockade of the reninangiotensin system (RAS).

A total of 414 patients were included in this cohort. The patients were divided into three groups according to their baseline urine albumin creatinine ratio (UACR): (1) a macroalbuminuria group (UACR $\geq 300 \mathrm{mg} / \mathrm{g}, n=38$ ), (2) a microalbuminuria group $(30 \mathrm{mg} / \mathrm{g} \leq \mathrm{UACR}<300 \mathrm{mg} / \mathrm{g}$, $n=116$ ), and (3) a normoalbuminuria group (UACR < $30 \mathrm{mg} / \mathrm{g}, n=260$ ). Additionally, we performed a long-term efficacy analysis to evaluate the effects of DPP-4i on the eGFR. Patients in the study cohort who had been prescribed DPP$4 \mathrm{i}$ continuously for more than 4 years and whose serum creatinine levels were measured at baseline, 4 years before and after the first prescription of DPP-4i, were included in the long-term efficacy analysis.

2.2. Data Extraction. The first prescription date of DPP$4 \mathrm{i}$ was defined as the index date, and the first prescribed DPP-4i was classified as the treatment drug in patients who were prescribed more than one DPP-4i. Cessation of DPP4i therapy was designated as the date of changing to another antidiabetic drug, a drug prescription gap of more than 30 days, or the study end date (May 31, 2015). Drug adherence was measured using the proportion of days covered (PDC, the days of taking the medicine divided by a whole followup duration). $\mathrm{PDC} \geq 0.80$ was considered to indicate drug adherence and patients with $\mathrm{PDC}<0.80$ were removed from the analyses.

Demographic characteristics, including age and gender, were extracted from index data. Blood pressure, height, weight, diabetes mellitus (DM) duration, and baseline laboratory tests-including $\mathrm{HbAlc}$, lipid profile, serum creatinine, urine creatinine, and urine albumin-were collected (i.e., the most recent values measured within 90-day range prior to the index date). Values for these parameters before and after treatment were also extracted using the same method. UACR was calculated using urine albumin and creatinine levels from an untimed spot urine collection. eGFR was measured using the Modification of Diet in Renal Disease Study Equation [23]:

$$
\begin{aligned}
& \mathrm{eGFR}=\left(186 \times(\text { serum creatinine })^{-1.154} \times(\text { Age })^{-0.203}\right. \\
& \quad \times(0.742 \text { if female }))
\end{aligned}
$$

2.3. Statistical Analysis. All analyses were performed using R software (ver. 3.2.3; R Development Core Team, Vienna, Austria). Data are expressed as means \pm standard deviation. A self-controlled design, in which comparisons are made within individuals, was used to estimate the renoprotective effect of DPP-4i. Using this method, all time-invariant confounders (e.g., sex, smoking, ethnicity, albuminuria status, other underlying diseases, and coadministrated drugs) were eliminated, and time-constant covariates (e.g., age, eGFR deterioration due to DM, and DM duration) were properly adjusted for. The paired Student's $t$-test was used to evaluate statistical differences between all parameters before and after DPP-4i treatment. Multiple linear regression analysis was performed to evaluate the effects of covariates on albuminuria reduction.

\section{Results}

3.1. Patients Characteristics. A total of 414 patients with T2DM satisfied the eligibility criteria of this study. The mean age of the included patients was $59.2 \pm 11.5$ years and the mean duration of DM was $11.0 \pm 7.4$ years. The mean BMI and $\mathrm{HbAlc}$ were $25.2 \pm 3.6 \mathrm{~kg} / \mathrm{m}^{2}$ and $8.6 \pm 1.5 \%$, respectively. Metformin and sulfonylurea were prescribed in $74.9 \%$ and $69.8 \%$ of patients, respectively, while $56.8 \%$ of patients were prescribed RAS inhibitors and $59.2 \%$ of patients were prescribed statins (Table 1).

\subsection{Changes in UACR and Metabolic Parameters 1 Year prior} to and 1 Year after DPP-4i Treatment. The mean UACR in all patients increased approximately $39 \mathrm{mg} / \mathrm{g}$ from 1 year before DPP-4i treatment to the point of DPP-4i treatment initiation, while it was decreased approximately $45 \mathrm{mg} / \mathrm{g} 1$ year after initiation of DPP-4i treatment $(p<0.05)$. Patients with macroalbuminuria $(\geq 300 \mathrm{mg} / \mathrm{g}$ ) showed significant reductions in albuminuria (Figure $1, p<0.05$ ); however, patients 


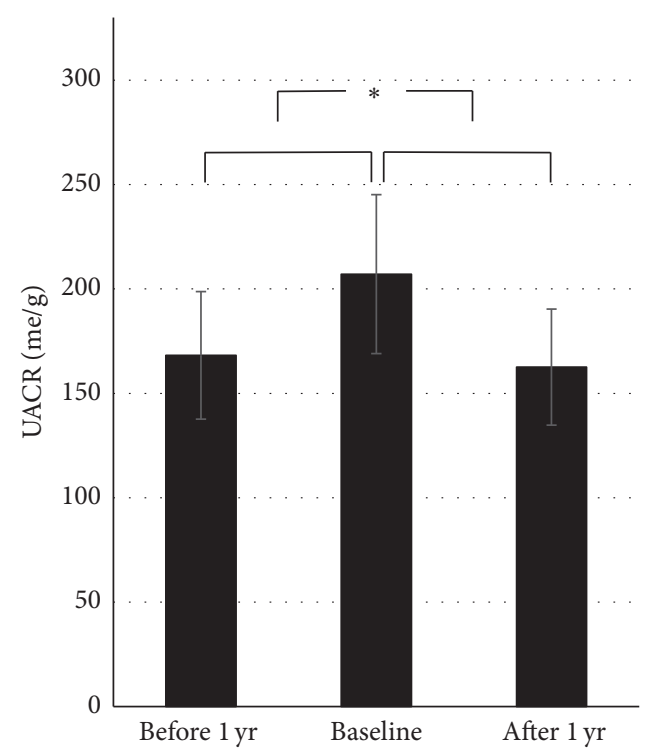

(a)

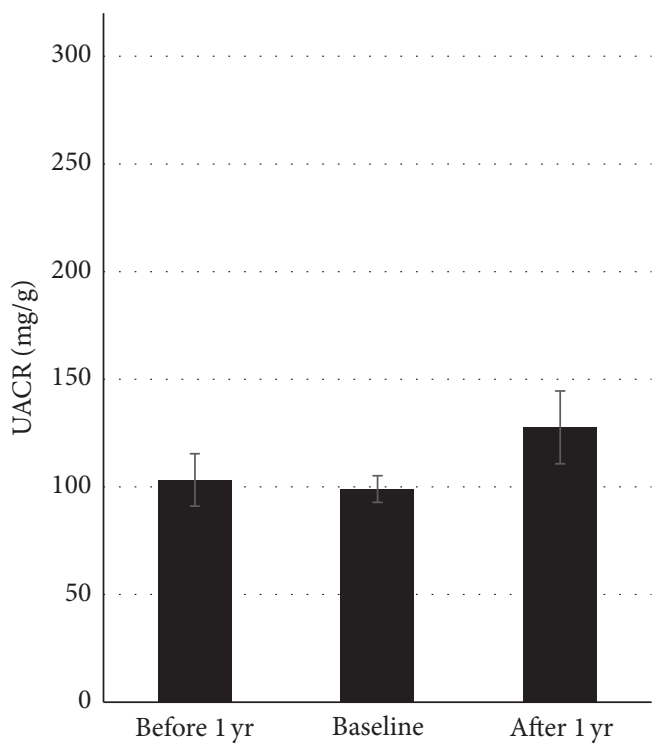

(c)

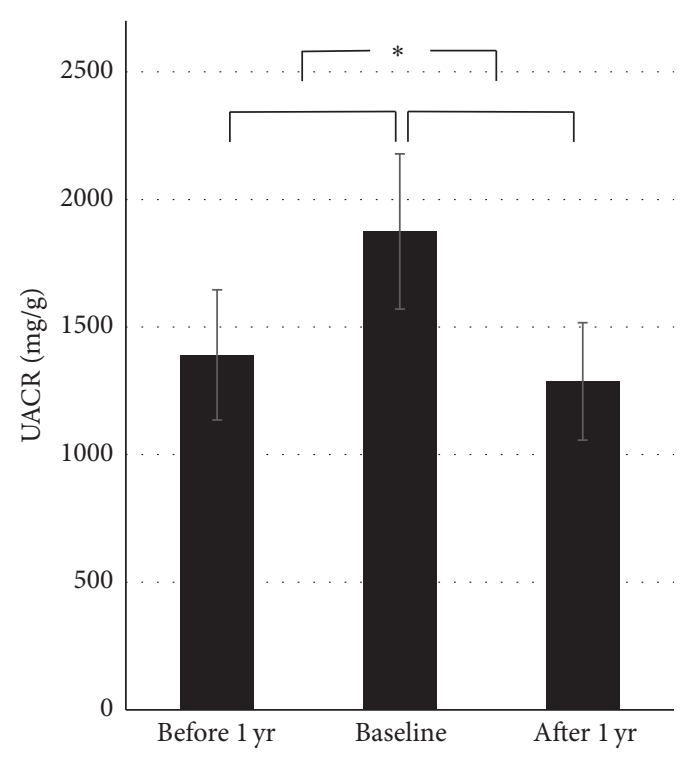

(b)

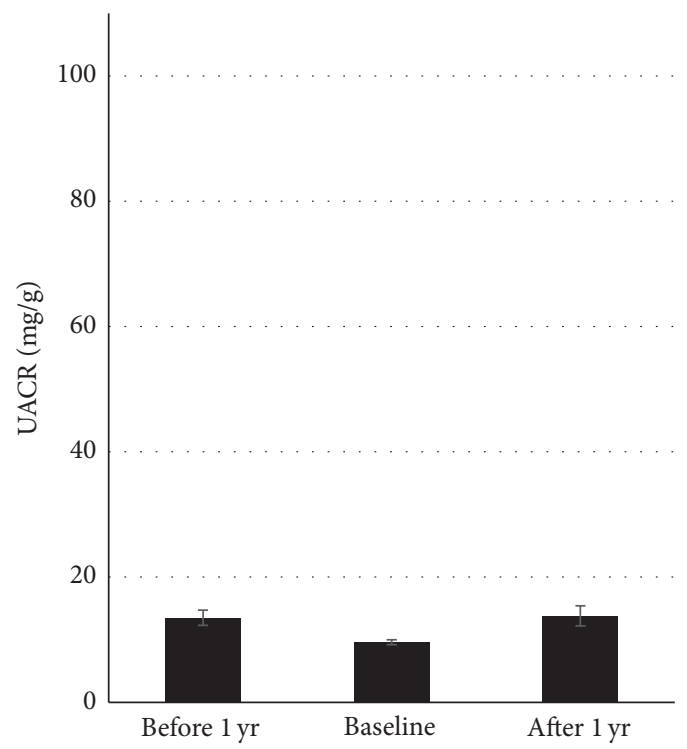

(d)

FIGURE 1: Changes in urine albumin/creatinine ratio 1 year before and 1 year after DPP-4i treatment initiation. Changes in urine albumin/creatinine ratio in all patients (a) and in patients with macroalbuminuria (b), microalbuminuria (c), and normoalbuminuria (d). (Data are presented as means with standard errors.) DPP-4i: dipeptidyl-peptidase IV inhibitor; UACR: urine albumin/creatinine ratio. ${ }^{*} p$ value $<0.05$.

with microalbuminuria and normoalbuminuria showed no significant changes.

The mean HbAlc improved from $8.6 \%(70 \mathrm{mmol} / \mathrm{mol})$ to $7.8 \%(62 \mathrm{mmol} / \mathrm{mol})(p<0.01)$, and the mean low-density lipoprotein- (LDL-) cholesterol level decreased from $89.8 \pm$ $39.5 \mathrm{mg} / \mathrm{dL}$ to $84.4 \pm 33.1 \mathrm{mg} / \mathrm{dL}$ ( $p<0.05)$. However, eGFR was not changed 1 year after DPP-4i treatment compared with 1 year before DPP-4i treatment (Table 2).

3.3. Estimating the Effect of Covariates on Albuminuria Reduction. To estimate the effect of covariates on albuminuria reduction, multiple linear regression analysis was performed. Although a reduction of HbAlc was shown using the paired Student's $t$-test, no significant decrease was seen on multiple linear regression analysis. Moreover, sex, age, and systolic blood pressure did not explain the changes seen in UACR on multiple linear regression analysis (Table 3).

3.4. Changes in eGFR 4 Years prior to and 4 Years after DPP$4 i$ Treatment. To verify the long-term effects of DPP-4i on eGFR, the change in eGFR from a point of treatment to 4 years before DPP- $4 \mathrm{i}$ treatment and 4 years after treatment 
TABLE 1: Patient baseline characteristics $(N=414)$.

\begin{tabular}{lc}
\hline Characteristics & Results \\
\hline Age (years) & $59.2 \pm 11.5$ \\
Sex $(n$, male/female) & $224 / 190$ \\
Body mass index $\left(\mathrm{kg} / \mathrm{m}^{2}\right)$ & $25.2 \pm 3.6$ \\
DM duration (years) & $11.0 \pm 7.4$ \\
Systolic blood pressure (mmHg) & $125.4 \pm 17.2$ \\
Diastolic blood pressure (mmHg) & $73.3 \pm 10.6$ \\
HbAlc (\%) & $8.6 \pm 1.5$ \\
LDL-cholesterol (mg/dL) & $89.8 \pm 39.5$ \\
HDL-cholesterol (mg/dL) & $46.4 \pm 12.0$ \\
Triglycerides (mg/dL) & $168.0 \pm 35.5$ \\
eGFR (mL/min/1.73 m ${ }^{2}$ ) & $68.3 \pm 17.6$ \\
Antidiabetic drugs (\%) & \\
$\quad$ Metformin & 74.9 \\
Sulfonylurea & 69.8 \\
Thiazolidinedione & 3.6 \\
$\quad$ Alpha-glucosidase inhibitor & 0.2 \\
RAS inhibitor (\%) & 56.8 \\
Statin (\%) & 59.2 \\
\hline
\end{tabular}

Data are presented as means \pm standard deviation or frequencies. eGFR: estimated glomerular filtration rate; HbAlc: glycosylated hemoglobin; HDL: high density lipoprotein; LDL: low density lipoprotein; RAS: reninangiotensin system.

was compared in patients who were prescribed DPP-4i for more than 4 years. A total of 78 patients were included in the analysis (characteristics of those patients were present in Supplementary Table 1 in Supplementary Material available online at http://dx.doi.org/10.1155/2016/1423191). The mean change in eGFR 4 years before treatment from baseline was $-22.4,-9.1$, and $-8.3 \mathrm{~mL} / \mathrm{min} / 1.73 \mathrm{~m}^{2}$ in the macroalbuminuria, microalbuminuria, and normoalbuminuria groups, respectively (Figure 2). However, 4 years after DPP- 4 i treatment initiation, the eGFR increased in the macroalbuminuria group from 54.3 to $58.5 \mathrm{~mL} / \mathrm{min} / 1.73 \mathrm{~m}^{2}$ and in the normoalbuminuria group from 70.3 to $77.5 \mathrm{~mL} / \mathrm{min} / 1.73 \mathrm{~m}^{2}$. In each group, paired Student's $t$-test on the eGFR change from a point of treatment to 4 years before DPP- $4 \mathrm{i}$ treatment and 4 years after treatment was statistically significant $(p<0.01$ for all groups).

3.5. Subgroup Analysis for Sex, Age, Obesity, Chronic Kidney Disease Stage, and Drug Coadministration. A subgroup analysis was performed to determine which subgroup was associated with UACR changes and what factors were associated with the albuminuria-lowering effect of DPP-4i. Albuminuria significantly decreased in patients $<65$ years old of both genders $(p<0.05)$. However, no significant differences in albuminuria were found when patients were divided according to their chronic kidney disease stage. Patients who were prescribed metformin, statins, and RAS inhibitors showed improvement in albuminuria $(p<0.05)$. Vildagliptin, sitagliptin, saxagliptin, and linagliptin decreased albuminuria without statistical significance (Table 4).

\section{Discussion}

This retrospective cohort study suggests that DPP-4i could reduce UACR, especially in T2DM patients with macroalbuminuria. Interestingly, DPP-4i reduced albuminuria in patients who were coadministered metformin or statins. Furthermore, DPP-4i could preserve eGFR in patients with T2DM, regardless of their baseline UACR.

Some mechanisms have been suggested to underlie the renoprotective effects of DPP- $4 \mathrm{i}$ in previous studies. DPP-4 shows the highest expression in the kidneys among all organs and is mainly expressed in the kidney proximal tubule in healthy humans [24]. However, in DM patients, DPP-4 is also present in the renal glomerulus [25]. DPP-4 inhibition by DPP-4i was shown to reduce kidney injury in rat models of diabetes $[16,17]$. One suggested mechanism underlying this effect is that DPP-4 inhibition upregulates renal cyclic adenosine monophosphate (cAMP) production by elevating circulatory stromal cell-derived factor-1a [26]. Increased cAMP has antioxidative effects and reduces reactive oxygen species, which are considered a major cause of diabetic nephropathy. Another suggested mechanism is that DPP-4i elevates active glucagon-like peptide-1, which is known to upregulate CAMP and reduce oxidative stress [27].

In our study, eGFR was increased in patients with macroalbuminuria or normoalbuminuria after taking DPP$4 \mathrm{i}(p<0.01)$, while the eGFR reduction rate in patients with microalbuminuria was slower during the 4 years after DPP-4i initiation relative to prior to treatment initiation $(p<$ 0.05). To our knowledge, this is the first study to show that DPP-4i alleviates eGFR decline. Previous studies have shown that eGFR was not improved after 3-6 months of DPP-4i treatment [20, 21]. Similarly, eGFR was not different 1 year after DPP-4i treatment but was improved after 4 years of treatment in our study. Thus, long-term DPP-4i treatment may mitigate the decline in eGFR associated with diabetic nephropathy or even improve it.

Urine albumin excretion was decreased after DPP-4i treatment in the macroalbuminuria group $(p<0.005)$ but not in the microalbuminuria and normoalbuminuria groups. This result is not consistent with previous studies, which suggested that DPP-4i had an albuminuria-lowering effect in patients with microalbuminuria $[18,21]$. However, the followup periods of the above-mentioned studies were shorter than that of our cohort (3-6 months) and their patients were prescribed only sitagliptin. Moreover, although HbAlc was decreased after DPP-4i administration, the effect of HbAlc on lowering albuminuria was not significant in multiple linear regression. The change in albuminuria was not influenced by sex, age, DM duration, eGFR, or systolic blood pressure at baseline.

In the subgroup analysis pertaining to coadministration of other drugs, urine albumin excretion was decreased in patients who were given metformin, statins, and RAS inhibitors. The renoprotective efficacy of metformin in T2DM patients remains controversial. Some studies insist that metformin lowers urine albumin excretion and has renoprotective effects $[28,29]$; however, other studies have shown that metformin did not exert these beneficial and 
TABLE 2: Changes in UACR, HbAlc, eGFR, and lipid profiles 1 year before and 1 year after DPP-4i treatment initiation.

\begin{tabular}{lccc}
\hline & $\begin{array}{c}\text { Changes during } \\
\text { 1 year before treatment }\end{array}$ & $\begin{array}{c}\text { Changes during } \\
\text { 1 year after treatment }\end{array}$ & $p$ value \\
\hline UACR $(\mathrm{mg} / \mathrm{g})$ & $40.8 \pm 307.8$ & $-44.5 \pm 351.9$ & $<0.05$ \\
HbAlc $(\%)$ & $0.4 \pm 1.1$ & $-0.8 \pm 1.5$ & $<0.01$ \\
Systolic blood pressure (mmHg) & $-1.2 \pm 20.5$ & $1.9 \pm 20.7$ & 0.13 \\
Diastolic blood pressure (mmHg) & $-0.3 \pm 12.8$ & $1.4 \pm 12.7$ & 0.37 \\
LDL-cholesterol (mg/dL) & $-1.2 \pm 26.32$ & $-3.5 \pm 30.2$ & $<0.05$ \\
HDL-cholesterol (mg/dL) & $-0.3 \pm 8.7$ & $-0.9 \pm 8.4$ & 0.30 \\
eGFR $\left(\mathrm{mL} / \mathrm{min} / 1.73 \mathrm{~m}^{2}\right)$ & $-0.7 \pm 8.7$ & $1.2 \pm 11.3$ & 0.69 \\
\hline
\end{tabular}

Data are presented as means \pm standard deviation.

${ }^{\ddagger}$ The paired Student's $t$-test was performed to evaluate changes in each parameter from baseline to 1 year before DPP-4i treatment and 1 year after treatment initiation.

DPP-4i: dipeptidyl-peptidase IV inhibitor; eGFR: estimated glomerular filtration rate; HbAlc: glycosylated hemoglobin; HDL: high density lipoprotein; LDL: low density lipoprotein; UACR: urine albumin/creatinine ratio.

TABLE 3: Multiple linear regression analysis for predictors of change of UACR.

\begin{tabular}{lcc}
\hline & $\beta$ & $p$ value \\
\hline Age & -0.002 & 0.78 \\
Sex (male) & 0.263 & 0.06 \\
Duration of diabetes & 0.010 & 0.18 \\
BMI & 0.003 & 0.77 \\
Systolic blood pressure & 0.006 & 0.12 \\
$\Delta$ HbAlc & 0.035 & 0.36 \\
LDDL-cholesterol & 0.001 & 0.63 \\
eGFR & 0.001 & 0.98 \\
\hline
\end{tabular}

BMI: body mass index; eGFR: estimated glomerular filtration rate; HbAlc: glycosylated hemoglobin; LDL: low-density lipoprotein.

protective effects [30, 31]. In our analysis, T2DM patients who were prescribed DPP-4i with metformin showed an improvement of albuminuria $(p<0.05)$. The combination of metformin and DPP-4i may have a synergistic renoprotective effect. However, a well-designed study with adequate power is warranted to verify this protective effect. Usually, statins disturb the uptake of plasma proteins in the glomerular tubule, which can result in albuminuria in some patients $[32,33]$. In our analysis, administration of DPP-4i in patients also taking statins resulted in improvements in urine albumin excretion. Physiologically, DPP-4 and the glucagonlike peptide-1 (which is the substrate of DPP4) receptor are localized in the renal tubule. DPP-4i may prevent statininduced proteinuria via unknown mechanisms, as well as decrease albuminuria caused by DM. However, our study was not specifically designed to evaluate any statin-induced proteinuria-lowering effect. Thus, large prospective cohort studies are needed to assess the synergistic effect of DPP-4i and statins on reduction of albuminuria.

An important strength of our study was that the cohort contained long-term treatment data. Previous studies investigating the renoprotective effects of DPP- 4 i used only shortterm data, and they showed only albuminuria-lowering effects $[18-21,34]$. Because our cohort contained patients' data 4 years prior to and 4 years following treatment initiation, we were able to demonstrate declines in eGFR being reduced and even reversed. A second strength of our study lies in the fact that we included five DPP-4i classes: sitagliptin, linagliptin, saxagliptin, vildagliptin, and gemigliptin. Although there were no significant differences between these drugs, the majority were associated with a reduction of albuminuria. It is possible that various DPP-4i classes exert different albuminuria-lowering effects in T2DM patients.

There were some limitations to our study. First, this study used a self-controlled design, as there was no control group. In this design, patient data prior to DPP-4i treatment were compared with data after DPP-4i treatment to estimate the effect of DPP-4i. Although there are some weaknesses in this design, time-invariant confounders and time-constant covariates were properly adjusted for using each patient's own data. Secondly, UACR was calculated from an untimed spot urine collection. A timed urine collection would better confirm albuminuria, as there are some diurnal variations and other conditions that affect creatinine excretion. However, timed urine collection is difficult under clinical circumstances because it is inconvenient. Unlike our retrospective study, a prospective cohort study, 


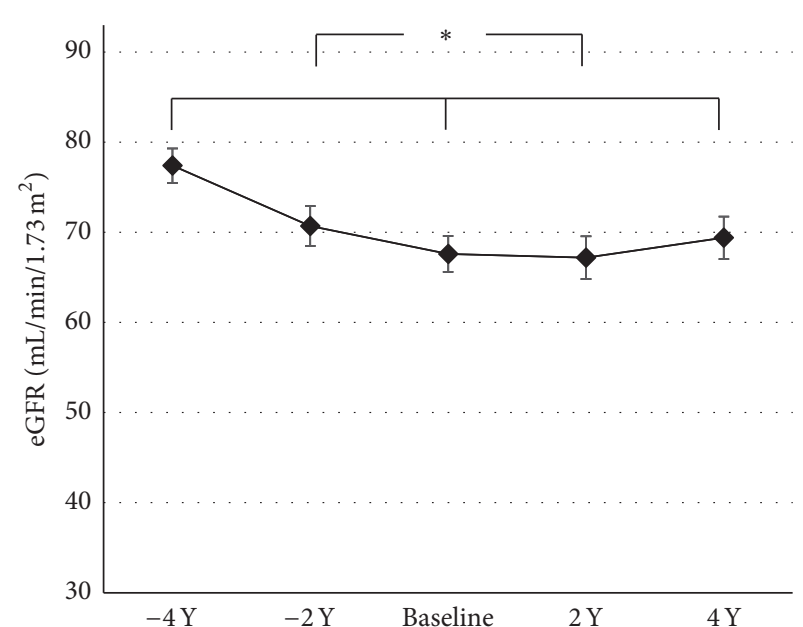

(a)

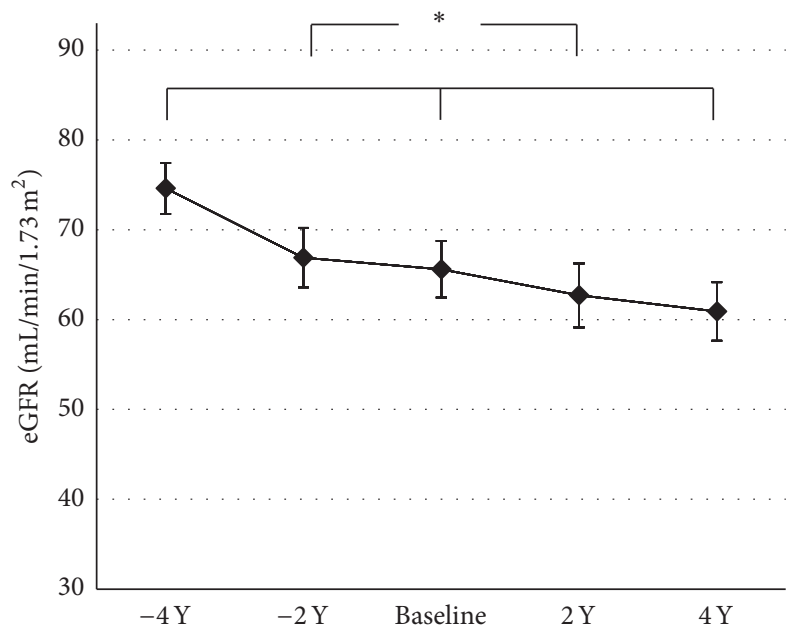

(c)

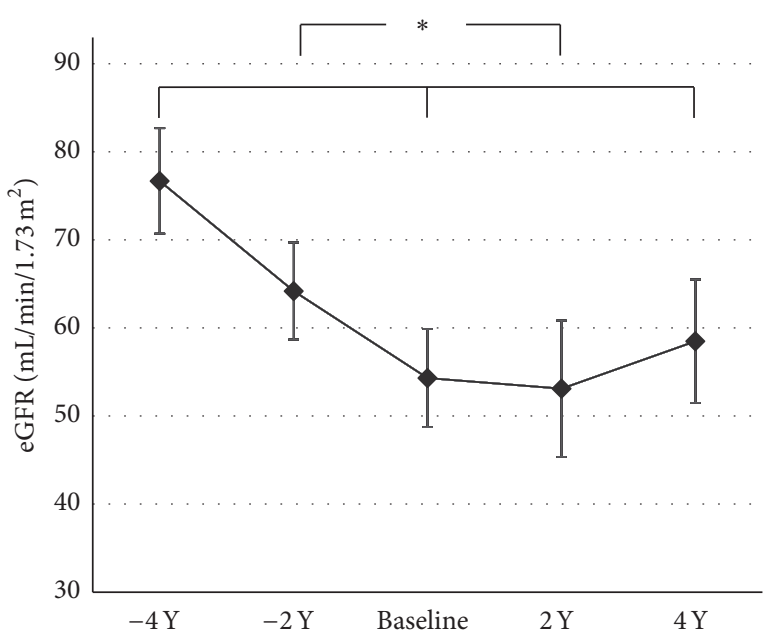

(b)

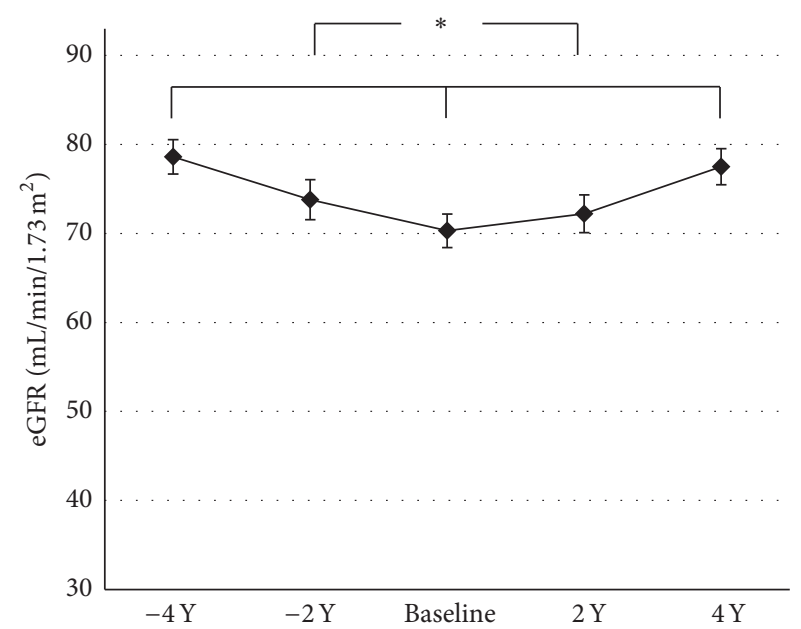

(d)

FIGURE 2: Changes in eGFR 4 years before and 4 years after DPP-4i treatment initiation. Changes in eGFR in all patients (a) and in patients with macroalbuminuria (b), microalbuminuria (c), and normoalbuminuria (d). Baseline values are the means with standard errors. DPP-4i: dipeptidyl-peptidase IV inhibitor; UACR: urine albumin/creatinine ratio; -4 Y: 4 years before DPP- 4 i treatment initiation; -2 Y: 2 years before DPP-4i treatment initiation; 2 Y: 2 years after DPP-4i treatment initiation; 4 Y: 4 years after DPP- 4 i treatment initiation. ${ }^{*} p$ value $<0.01$.

like MARLINA-T2D study, will be able to circumvent such disadvantages [19].

In conclusion, the present study demonstrated that DPP-4i treatment could ameliorate diabetic nephropathy, by reducing urine albumin excretion and mitigating the reduction of eGFR in T2DM patients.

\section{Competing Interests}

The authors declare no competing interests.

\section{Authors' Contributions}

Young-Gun Kim and Jung Hyun Byun contributed equally to this work as co-first authors. Young-Gun Kim designed the study, analyzed data, and wrote the manuscript. JungHyun
Byun and Dukyong Yoon collected the data and analyzed data. Ja Young Jeon, Seung Jin Han, Dae Jung Kim, and KwanWoo Lee contributed to the discussion and reviewed and edited the manuscript. Rae Woong Park and Hae Jin Kim designed the study and wrote the manuscript.

\section{Acknowledgments}

This work was supported by the Basic Science Research Program through the National Research Foundation of Korea (NRF) funded by the Ministry of Science, ICT \& Future Planning (Grant no. NRF-2014R1A1A3050777); the Korea Health Technology R\&D Project through the Korea Health Industry Development Institute (KHIDI), funded by the Ministry of Health \& Welfare, Republic of Korea (Grants nos. HI16C0992 and HI14C3201). 
TABLE 4: Subgroup analysis for sex, age, obesity, chronic kidney disease stage, and drug coadministration.

\begin{tabular}{|c|c|c|c|c|c|c|c|}
\hline & $N$ & $\begin{array}{c}\text { UACR } \\
1 \text { year before } \\
\text { treatment }\end{array}$ & Baseline UACR & $\begin{array}{c}\text { UACR } \\
1 \text { year after } \\
\text { treatment }\end{array}$ & $\begin{array}{c}\text { UACR change } \\
\text { during } \\
1 \text { year before } \\
\text { treatment }\end{array}$ & $\begin{array}{c}\text { UACR change } \\
\text { during } \\
1 \text { year after } \\
\text { treatment }\end{array}$ & $p$ value $^{\ddagger}$ \\
\hline \multicolumn{8}{|l|}{ Sex } \\
\hline Male & 224 & $127.2 \pm 401.4$ & $168.5 \pm 541.8$ & $135.6 \pm 398.1$ & $41.3 \pm 227.3$ & $-32.9 \pm 282.6$ & $<0.05$ \\
\hline Female & 190 & $212.4 \pm 802.2$ & $252.5 \pm 979.7$ & $194.3 \pm 714.3$ & $40.1 \pm 380.4$ & $-58.2 \pm 419.5$ & $<0.05$ \\
\hline \multicolumn{8}{|l|}{ Age } \\
\hline$\geq 65$ years & 133 & $153.2 \pm 384.0$ & $166.0 \pm 551.2$ & $151.7 \pm 484.5$ & $12.8 \pm 231.3$ & $-14.3 \pm 273.4$ & 0.66 \\
\hline$<65$ years & 281 & $171.0 \pm 715.1$ & $226.5 \pm 860.1$ & $167.7 \pm 600.9$ & $55.5 \pm 340.9$ & $-58.8 \pm 382.8$ & $<0.05$ \\
\hline \multicolumn{8}{|l|}{ Obesity } \\
\hline Obese & 151 & $162.0 \pm 654.5$ & $232.5 \pm 869.5$ & $172.3 \pm 585.1$ & $70.5 \pm 437.2$ & $-60.2 \pm 433.2$ & 0.24 \\
\hline Nonobese & 159 & $206.0 \pm 770.5$ & $256.8 \pm 887.4$ & $220.6 \pm 691.7$ & $50.8 \pm 267.1$ & $-36.2 \pm 348.6$ & 0.10 \\
\hline \multicolumn{8}{|l|}{$C K D$} \\
\hline $\mathrm{eGFR} \geq 90$ & 41 & $131.2 \pm 231.1$ & $126.6 \pm 491.6$ & $62.2 \pm 158.0$ & $-4.6 \pm 67.3$ & $-64.4 \pm 419.3$ & 0.92 \\
\hline $90>\mathrm{eGFR} \geq 60$ & 229 & $91.1 \pm 496.3$ & $104.6 \pm 524.7$ & $81.6 \pm 368.7$ & $13.5 \pm 149.0$ & $-23.0 \pm 229.9$ & 0.29 \\
\hline $60>\mathrm{eGFR} \geq 30$ & 115 & $306.2 \pm 652.8$ & $338.2 \pm 864.5$ & $286.7 \pm 716.5$ & $32 \pm 261.5$ & $-51.5 \pm 383.8$ & 0.11 \\
\hline $30>\mathrm{eGFR} \geq 15$ & 13 & $861.8 \pm 1631.2$ & $917.6 \pm 2446.1$ & $886.9 \pm 1599.0$ & $55.8 \pm 1181.9$ & $-30.7 \pm 1074.0$ & 0.27 \\
\hline \multicolumn{8}{|l|}{ Metformin } \\
\hline Yes & 310 & $156.0 \pm 573.2$ & $187.5 \pm 678.2$ & $137.0 \pm 508.3$ & $21.9 \pm 193.7$ & $-50.5 \pm 319.8$ & $<0.05$ \\
\hline No & 104 & $174.6 \pm 737.5$ & $265.3 \pm 1009.5$ & $238.7 \pm 707.0$ & $90.7 \pm 495.1$ & $-26.6 \pm 435.0$ & 0.25 \\
\hline \multicolumn{8}{|l|}{ Sulfonylurea } \\
\hline Yes & 289 & $170.4 \pm 401.0$ & $179.0 \pm 560.8$ & $153.7 \pm 465.1$ & $8.6 \pm 171.4$ & $-25.3 \pm 310.1$ & 0.46 \\
\hline No & 125 & $130.9 \pm 1039.6$ & $271.9 \pm 1122.5$ & $182.9 \pm 750.1$ & $141 \pm 537.1$ & $-89 \pm 431.5$ & $<0.05$ \\
\hline \multicolumn{8}{|l|}{ Statin } \\
\hline Yes & 245 & $201.8 \pm 752.9$ & $259.6 \pm 933.4$ & $203.5 \pm 687.3$ & $57.8 \pm 382.9$ & $-56.1 \pm 398.3$ & $<0.05$ \\
\hline No & 169 & $114.5 \pm 352.9$ & $131.0 \pm 445.5$ & $103.3 \pm 307.2$ & $16.5 \pm 142.1$ & $-27.7 \pm 271.1$ & 0.54 \\
\hline \multicolumn{8}{|l|}{ RAS inhibitor } \\
\hline Yes & 235 & $238.9 \pm 765.7$ & $299.3 \pm 941.2$ & $219.1 \pm 645.9$ & $60.4 \pm 383.0$ & $-80.2 \pm 438.8$ & $<0.05$ \\
\hline No & 179 & $73.3 \pm 289.1$ & $86.0 \pm 447.5$ & $88.4 \pm 429.2$ & $12.7 \pm 140.7$ & $2.4 \pm 173.9$ & 0.95 \\
\hline \multicolumn{8}{|l|}{$D P P-4 i$} \\
\hline Vildagliptin & 136 & $185.6 \pm 779.5$ & $245.1 \pm 855.5$ & $191.2 \pm 618.5$ & $59.5 \pm 266.2$ & $-53.8 \pm 257.1$ & 0.08 \\
\hline Sitagliptin & 96 & $208.0 \pm 181.3$ & $209.0 \pm 590.3$ & $143.9 \pm 349.2$ & $1.0 \pm 88.3$ & $-65.1 \pm 479.4$ & 0.66 \\
\hline Linagliptin & 77 & $144.8 \pm 776.5$ & $243.2 \pm 1073.1$ & $200.4 \pm 724.2$ & $98.4 \pm 54.3$ & $-42.8 \pm 45.3$ & 0.30 \\
\hline Saxagliptin & 56 & $233.7 \pm 530.9$ & $221.1 \pm 688.9$ & $188.8 \pm 709.8$ & $-12.6 \pm 206.5$ & $-32.3 \pm 287.4$ & 0.20 \\
\hline Gemigliptin & 48 & $3.3 \pm 27.1$ & $7.2 \pm 44.8$ & $12.3 \pm 63.6$ & $3.9 \pm 28.8$ & $5.1 \pm 32.0$ & 0.60 \\
\hline
\end{tabular}

Data are presented as means \pm standard deviation.

Some patients were not included in the subgroup analyses due to missing data.

CKD: chronic kidney disease; DPP-4i: dipeptidyl-peptidase IV inhibitor; eGFR: estimated glomerular filtration rate; RAS: renin-angiotensin-system; UACR: urine albumin/creatinine ratio.

\section{References}

[1] A. I. Adler, R. J. Stevens, S. E. Manley, R. W. Bilous, C. A. Cull, and R. R. Holman, "Development and progression of nephropathy in type 2 diabetes: The United Kingdom Prospective Diabetes Study (UKPDS 64)," Kidney International, vol. 63, no. 1, pp. 225-232, 2003.

[2] K. R. Tuttle, G. L. Bakris, R. W. Bilous et al., "Diabetic kidney disease: a report from an ADA consensus conference," Diabetes Care, vol. 37, no. 10, pp. 2864-2883, 2014.
[3] K. Matsushita, M. van der Velde, B. C. Astor et al., "Association of estimated glomerular filtration rate and albuminuria with all-cause and cardiovascular mortality in general population cohorts: a collaborative meta-analysis," The Lancet, vol. 375, no. 9731, pp. 2073-2081, 2010.

[4] R. T. Gansevoort, K. Matsushita, M. van der Velde et al., "Lower estimated GFR and higher albuminuria are associated with adverse kidney outcomes. A collaborative meta-analysis of general and high-risk population cohorts," Kidney International, vol. 80, no. 1, pp. 93-104, 2011. 
[5] M. Van Der Velde, K. Matsushita, J. Coresh et al., "Lower estimated glomerular filtration rate and higher albuminuria are associated with all-cause and cardiovascular mortality. A collaborative meta-analysis of high-risk population cohorts," Kidney International, vol. 79, no. 12, pp. 1341-1352, 2011.

[6] B. C. Astor, K. Matsushita, R. T. Gansevoort et al., "Lower estimated glomerular filtration rate and higher albuminuria are associated with mortality and end-stage renal disease. A collaborative meta-analysis of kidney disease population cohorts," Kidney International, vol. 79, no. 12, pp. 1331-1340, 2011.

[7] D. De Zeeuw, G. Remuzzi, H.-H. Parving et al., "Proteinuria, a target for renoprotection in patients with type 2 diabetic nephropathy: lessons from RENAAL," Kidney International, vol. 65, no. 6, pp. 2309-2320, 2004.

[8] M. A. Pohl, S. Blumenthal, D. J. Cordonnier et al., "Independent and additive impact of blood pressure control and angiotensin II receptor blockade on renal outcomes in the irbesartan diabetic nephropathy trial: clinical implications and limitations," Journal of the American Society of Nephrology, vol. 16, no. 10, pp. 30273037, 2005.

[9] W. B. A. Eijkelkamp, Z. Zhang, G. Remuzzi et al., "Albuminuria is a target for renoprotective therapy independent from blood pressure in patients with type 2 diabetic nephropathy: post hoc analysis from the reduction of endpoints in NIDDM with the angiotensin II antagonist losartan (RENAAL) trial," Journal of the American Society of Nephrology, vol. 18, no. 5, pp. 1540-1546, 2007.

[10] L. F. Fried and J. Lewis, "Albuminuria is not an appropriate therapeutic target in patients with CKD: the con view, Clinical Journal of the American Society of Nephrology, vol. 10, no. 6, pp. 1089-1093, 2015.

[11] V. L. M. Esnault, A. Ekhlas, C. Delcroix, M.-G. Moutel, and J.M. Nguyen, "Diuretic and enhanced sodium restriction results in improved antiproteinuric response to RAS blocking agents," Journal of the American Society of Nephrology, vol. 16, no. 2, pp. 474-481, 2005.

[12] L. Vogt, F. Waanders, F. Boomsma, D. De Zeeuw, and G. Navis, "Effects of dietary sodium and hydrochlorothiazide on the antiproteinuric efficacy of losartan," Journal of the American Society of Nephrology, vol. 19, no. 5, pp. 999-1007, 2008.

[13] E. K. Jackson, S. J. Kochanek, and D. G. Gillespie, "Dipeptidyl peptidase IV regulates proliferation of preglomerular vascular smooth muscle and mesangial cells," Hypertension, vol. 60, no. 3, pp. 757-764, 2012.

[14] R. Mentlein, "Dipeptidyl-peptidase IV (CD26)-role in the inactivation of regulatory peptides," Regulatory Peptides, vol. 85, no. 1, pp. 9-24, 1999.

[15] Y. Kirino, Y. Sato, T. Kamimoto, K. Kawazoe, K. Minakuchi, and Y. Nakahori, "Interrelationship of dipeptidyl peptidase IV (DPP4) with the development of diabetes, dyslipidaemia and nephropathy: a streptozotocin-induced model using wild-type and DPP4-deficient rats," Journal of Endocrinology, vol. 200, no. 1, pp. 53-61, 2009.

[16] W. J. Liu, S. H. Xie, Y. N. Liu et al., "Dipeptidyl peptidase IV inhibitor attenuates kidney injury in streptozotocin-induced diabetic rats," The Journal of Pharmacology and Experimental Therapeutics, vol. 340, no. 2, pp. 248-255, 2012.

[17] C. Mega, E. T. de Lemos, H. Vala et al., "Diabetic nephropathy amelioration by a low-dose sitagliptin in an animal model of type 2 diabetes (Zucker diabetic fatty rat)," Experimental Diabetes Research, vol. 2011, Article ID 162092, 12 pages, 2011.
[18] S. Hattori, "Sitagliptin reduces albuminuria in patients with type 2 diabetes," Endocrine Journal, vol. 58, no. 1, pp. 69-73, 2011.

[19] P.-H. Groop, M. E. Cooper, V. Perkovic et al., "Dipeptidyl peptidase-4 inhibition with linagliptin and effects on hyperglycaemia and albuminuria in patients with type 2 diabetes and renal dysfunction: rationale and design of the MARLINAT2D ${ }^{\mathrm{TM}}$ trial," Diabetes and Vascular Disease Research, vol. 12, no. 6, pp. 455-462, 2015.

[20] H. Mori, Y. Okada, T. Arao, and Y. Tanaka, "Sitagliptin improves albuminuria in patients with type 2 diabetes mellitus," Journal of Diabetes Investigation, vol. 5, no. 3, pp. 313-319, 2014.

[21] I. Kawasaki, Y. Hiura, A. Tamai et al., "Sitagliptin reduces the urine albumin-to-creatinine ratio in type 2 diabetes through decreasing both blood pressure and estimated glomerular filtration rate," Journal of Diabetes, vol. 7, no. 1, pp. 41-46, 2015.

[22] I. Goldshtein, A. Karasik, C. Melzer-Cohen et al., "Urinary albumin excretion with sitagliptin compared to sulfonylurea as add on to metformin in type 2 diabetes patients with albuminuria: a real-world evidence study," Journal of Diabetes and Its Complications, vol. 30, no. 7, pp. 1354-1359, 2016.

[23] A. S. Levey, J. Coresh, T. Greene et al., "Expressing the modification of diet in renal disease study equation for estimating glomerular filtration rate with standardized serum creatinine values," Clinical Chemistry, vol. 53, no. 4, pp. 766-772, 2007.

[24] P. Schlatter, C. Beglinger, J. Drewe, and H. Gutmann, "Glucagon-like peptide 1 receptor expression in primary porcine proximal tubular cells," Regulatory Peptides, vol. 141, no. 1-3, pp. 120-128, 2007.

[25] Y. Sharkovska, C. Reichetzeder, M. Alter et al., "Blood pressure and glucose independent renoprotective effects of dipeptidyl peptidase- 4 inhibition in a mouse model of type- 2 diabetic nephropathy," Journal of Hypertension, vol. 32, no. 11, pp. 22112223, 2014.

[26] H. Fujita, H. Taniai, H. Murayama et al., "DPP-4 inhibition with alogliptin on top of angiotensin II type 1 receptor blockade ameliorates albuminuria via up-regulation of SDF- $1 \alpha$ in type 2 diabetic patients with incipient nephropathy," Endocrine Journal, vol. 61, no. 2, pp. 159-166, 2014.

[27] G. G. Holz, "Epac: a new cAMP-binding protein in support of glucagon-like peptide-1 receptor-mediated signal transduction in the pancreatic $\beta$-cell," Diabetes, vol. 53, no. 1, pp. 5-13, 2004.

[28] N. Amador-Licona, J. M. Guízar-Mendoza, E. Vargas, G. Sánchez-Camargo, and L. Zamora-Mata, "The short-term effect of a switch from glybenclamide to metformin on blood pressure and microalbuminuria in patients with type 2 diabetes mellitus," Archives of Medical Research, vol. 31, no. 6, pp. 571-575, 2000.

[29] H. Nasri, A. Baradaran, M. R. Ardalan, S. Mardani, A. Momeni, and M. Rafieian-Kopaei, "Bright renoprotective properties of metformin: beyond blood glucose regulatory effects," Iranian Journal of Kidney Diseases, vol. 7, no. 6, pp. 423-428, 2013.

[30] J. M. Lachin, G. Viberti, B. Zinman et al., "Renal function in type 2 diabetes with rosiglitazone, metformin, and glyburide monotherapy," Clinical Journal of the American Society of Nephrology, vol. 6, no. 5, pp. 1032-1040, 2011.

[31] G. Schernthaner, D. R. Matthews, B. Charbonnel, M. Hanefeld, and P. Brunetti, "Efficacy and safety of pioglitazone versus metformin in patients with type 2 diabetes mellitus: a doubleblind, randomized trial," The Journal of Clinical Endocrinology \& Metabolism, vol. 89, no. 12, pp. 6068-6076, 2004.

[32] J. P. Deslypere, J. Delanghe, and A. Vermeulen, "Proteinuria as complication of simvastatin treatment," The Lancet, vol. 336, no. 8728, p. 1453, 1990. 
[33] A. Verhulst, P. C. D'Haese, and M. E. De Broe, "Inhibitors of HMG-CoA reductase reduce receptor-mediated endocytosis in human kidney proximal tubular cells," Journal of the American Society of Nephrology, vol. 15, no. 9, pp. 2249-2257, 2004.

[34] S. Tani, K. Nagao, and A. Hirayama, "Association between urinary albumin excretion and low-density lipoprotein heterogeneity following treatment of type 2 diabetes patients with the dipeptidyl peptidase- 4 inhibitor, vildagliptin: a pilot study," American Journal of Cardiovascular Drugs, vol. 13, no. 6, pp. 443-450, 2013. 


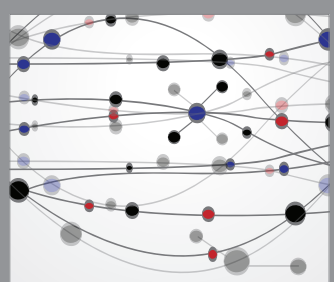

The Scientific World Journal
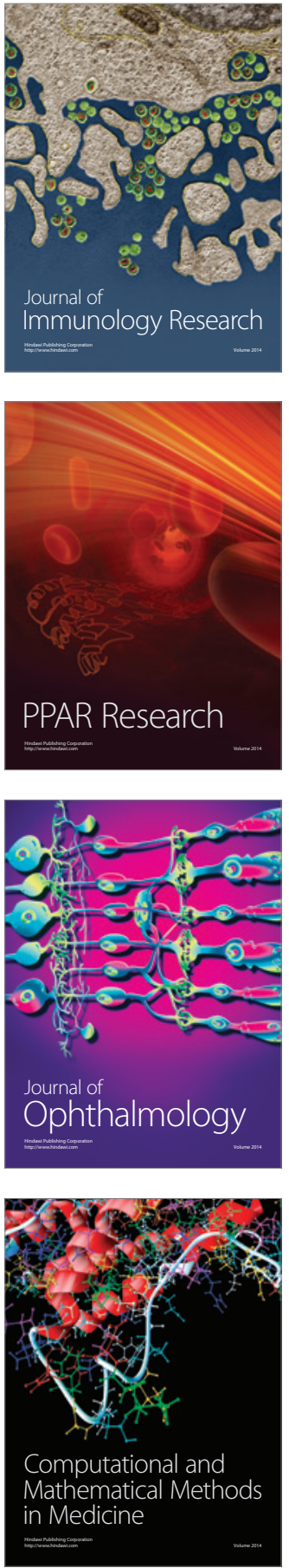

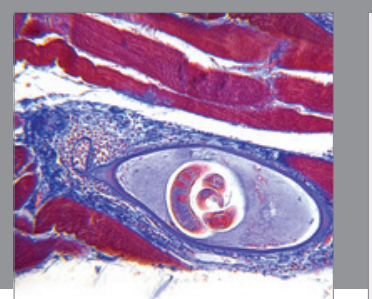

Gastroenterology Research and Practice

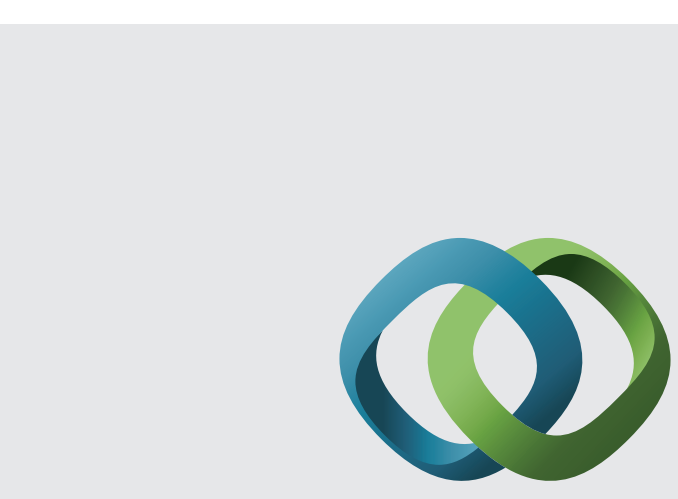

\section{Hindawi}

Submit your manuscripts at

http://www.hindawi.com
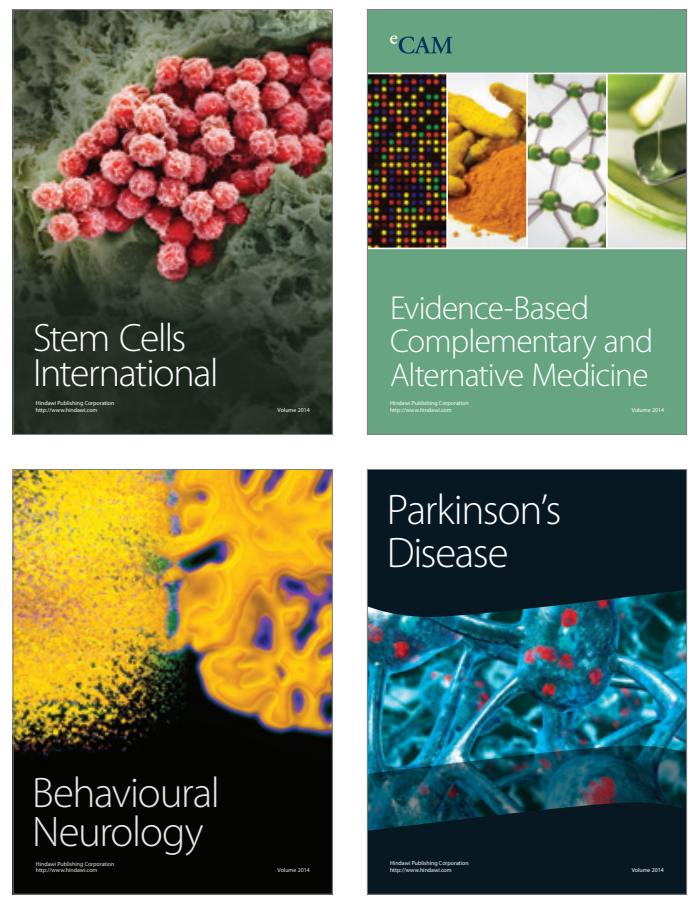
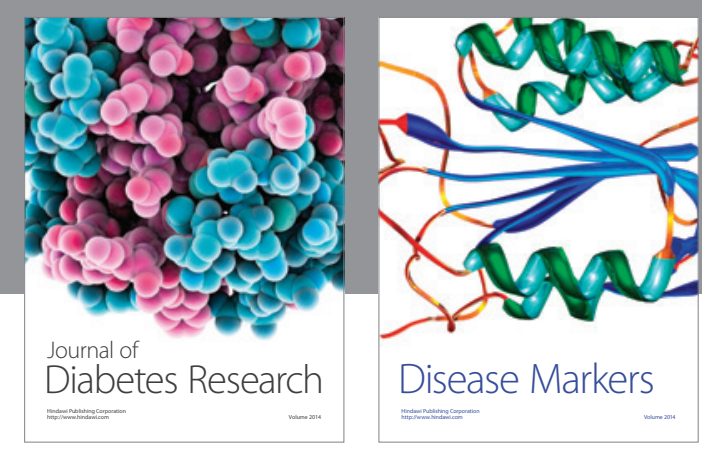

Disease Markers
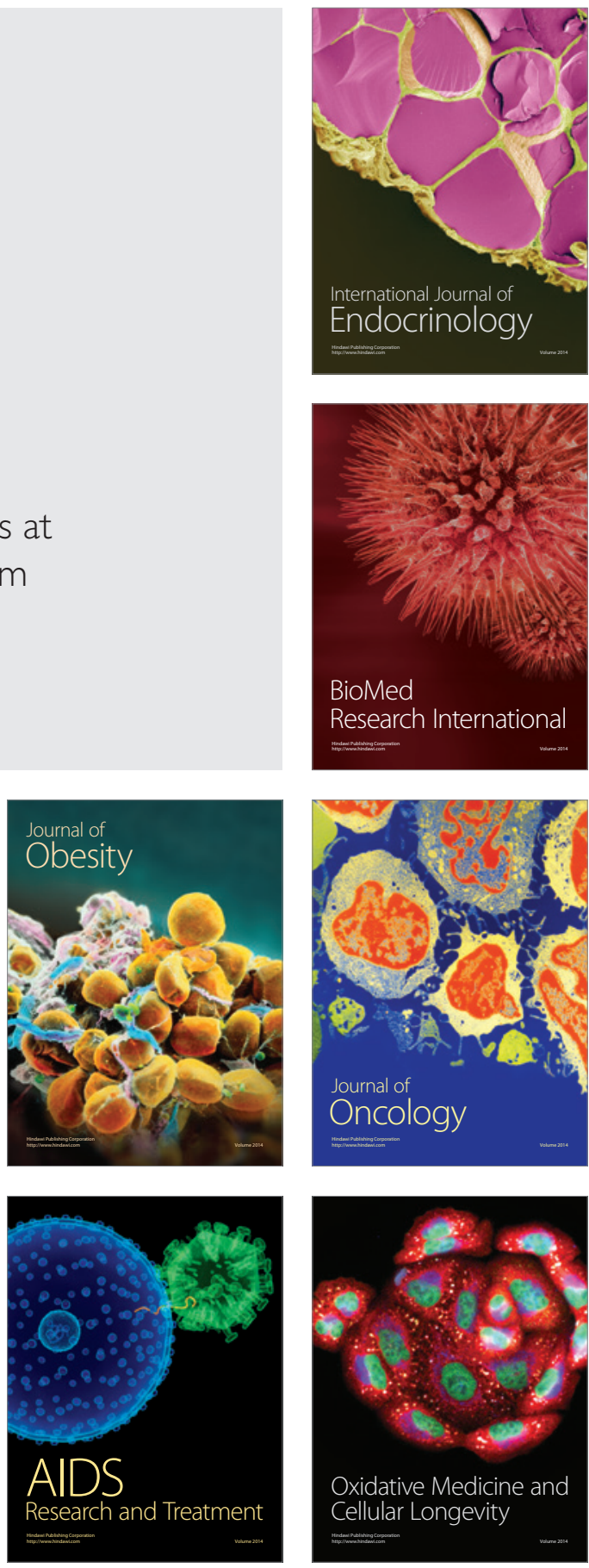